\title{
CORRIGENDA
}

to

\section{UNIFORM DISTRIBUTION AND LATTICE POINT COUNTING}

\author{
G. R. EVEREST
}

(Received 2 June 1992)

Equation (38) of this paper (J. Austral. Math. Soc. 53 (1992),39-50) is incorrect. It should read,

$$
U(q)=\frac{\omega_{K}(r+1)^{r}}{R_{K} r !}(\log q)^{r}+o\left((\log q)^{r-1}\right) .
$$

Also, the formula in Note 1 after Theorem 4 should read

$$
\sum_{i=1}^{r+1} u_{i}=0 .
$$

The reason for the error is that the behaviour of the function $I(z)$ at $z=r-1$ was wrongly calculated to be simple polar. In fact $I(z)$ is analytic away from $z=r$, as we now demonstrate.

Replace each of the $\zeta_{i}(y)$ by $2 \zeta_{i}(y)$ and denote the resulting integral by $I_{2}(z)$. Obviously $I_{2}(z)=2^{-z} I(z)$. Alternatively, write $2 \zeta_{i}(y)=\zeta_{i}(2 y)$ and change the variable $2 y=y^{\prime}$. The result is to multiply by $2^{-r}$, and to alter the compact ball $F$. But we remarked that changing the ball only adds an entire function to the integral. Thus, the residue of $I_{2}(z)$ at any pole, $z=z_{0}$, differs from that of $I(z)$ by $2^{-z_{0}}$ and $2^{-r}$. This shows that $z=r$ is the only pole.

School of Mathematics

University of East Anglia

Norwich NR4 7TJ

England

(C) 1994 Australian Mathematical Society 0263-61 15/94 \$A2.00+0.00 